\title{
PHOTO-PATTERNABLE GELATIN AS PROTECTION LAYERS IN SURFACE MICROMACHININGS
}

\author{
Lung-Jieh Yang, Wei-Zhi Lin, *Tze-Jung Yao and *Yu-Chong Tai \\ Dept. of Mechanical Engineering, Tamkang University, 151, Ying-Chuan Rd., Tamsui, 25137, Taiwan ROC \\ *Dept. of Electrical Engineering, California Institute of Technology, 136-93, Caltech, Pasadena, CA 91125, USA \\ Phone:+886-2-26215656 ext.2768, Fax:+886-2-26209745, E-mail: ljyang@mail.tku.edu.tw
}

\begin{abstract}
This paper describes a newly developed low-temperature photo-patternable Gelatin technology that is useful to produce a thick (greater than 10 microns) Gelatin protecting and strengthening layer for weak MEMS micro- structures. Example demonstrated here is the Gelatin process integrated with the Parylene MEMS technology. What is reported here is the complete processing details and formulae that allow anyone to use Gelatin like photo-resist. We find that it is a chemical-resistant and mechanical-robust material for MEMS applications.
\end{abstract}

\section{INTRODUCTION}

In the recent development of MEMS technology, people look for new processing and new materials with the characteristics of low-temperature. Lower processing-temperature makes the fabrication more feasible and easier in the complicated integration of MEMS products. The processing temperature (of structural layers) below $100^{\circ} \mathrm{C}$ often means the good chance of using photo-resist as the sacrificial layer in the surface micromachining. This is why the Parylene MEMS technology attracted much attention in the recent years [1] [2].

For the conventional fabrication of Parylene surface-micromachined diaphragms or wide channels, however, we usually adopt the design of supporting dimples or supercritical drying method to prevent MEMS stiction or collapse. The former modifies and changes the geometry of microstructures from the original designs and hence may cause undesirable effects. The latter has its problem of delaminating the Parylene structural layers from the substrate due to the penetration of supercritical $\mathrm{CO}_{2}$ through Parylene films. Therefore, developing a new kind of strengthening layer with low processing temperature to prevent the stiction issue of Parylene technology is needed. In this paper, we find that the Gelatin films can solve the problem mentioned above.

Gelatin has been used for over 6,000 years. It is made of proteins ( 18 kinds of amino acids) and is regarded as a pure food in the past. The most well known example is the "gummy bear" shown in Fig.1.

The low melting point of Gelatin $\left(<100^{\circ} \mathrm{C}\right)$, as indicated in Fig. 2, allows it to be formed into any shape by gentle heating/cooking [3].

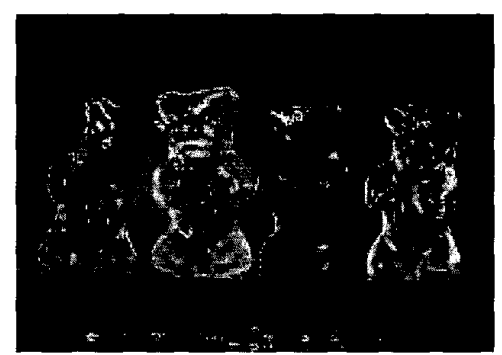

Fig. 1 Gummy bears [1]

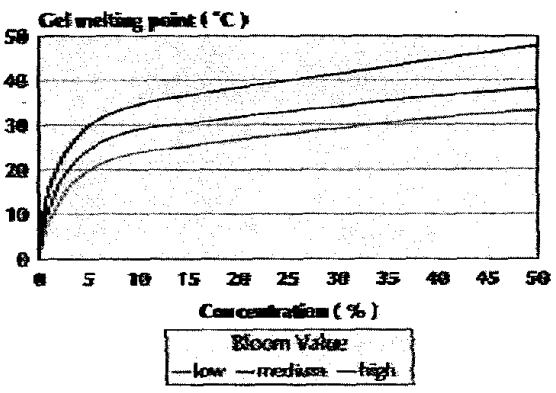

Fig. 2 Melting points of Gelatin [1]

Interestingly, Gelatin can be used as a binder in light-sensitive photolithographic products. Adding some dichromate into Gelatin makes it a photo-patternable material like negative photo-resist. That is, the portion of Gelatin exposing to the ultra-violet light becomes cross-linked and has a high selectivity to the warm water. In the following section, we demonstrate how to prepare the photo-sensitive Gelatin films by the popular spin-coating and photo-lithography. 


\section{PREPARATION OF GELATIN PATTERNS}

Before the spin-coating process, the Gelatin is photosensitized by the addition of dichromate ion. A typical formulation is Gelatin powder dissolved in warm water with 0.75 weight percent of potassium dichromate $\left(\mathrm{K}_{2} \mathrm{Cr}_{2} \mathrm{O}_{7}\right)$. This solution is normally spin-coated on the substrate, but the on-site IR heating is required if the Gelatin concentration is denser than $10 \%$. Room temperature drying of the coated film lasts for one day before the photolithography. Moderate exposure time of UV light should be tried and settled according to different thickness. Finally, removing uncrossed-linked Gelatin in water at $80^{\circ} \mathrm{C}$ develops the film. Figure 3 shows the thickness of cross-linked Gelatin film subject to different coating speed and concentration. Basically, it is difficult to have Gelatin film thicker than 1 microns with the concentration diluter than $10 \%$. Figures 4,5 , and 6 demonstrate several topological views of cross-linked Gelatin subjected to different concentration and line-width resolution.

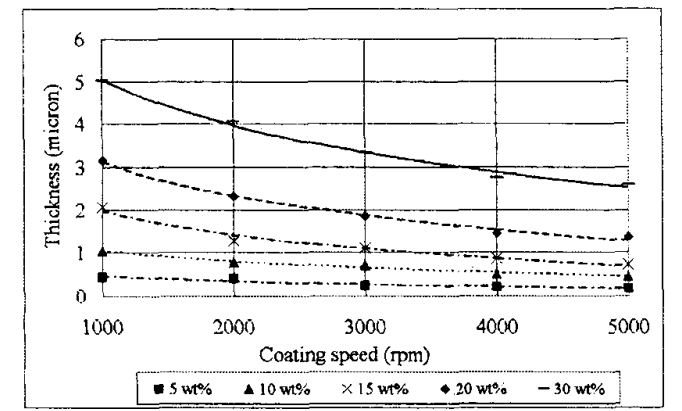

Fig. 3 The Thickness of Gelatin film vs. coating speed corresponding to different concentrations

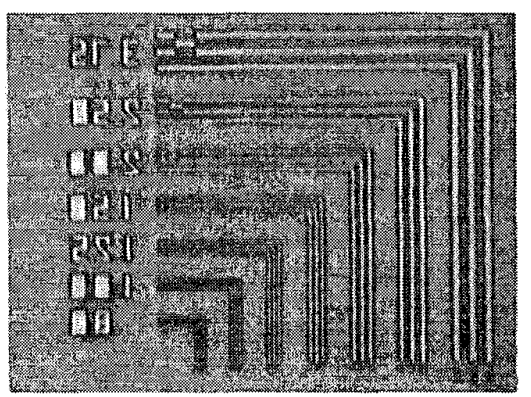

Fig. 4 Cross-linked Gelatin (4\%); thickness: 0.2 micron; minimum line-width 1 micron

\section{PROPERTIES OF GELATIN FILM}

Mechanically, Gelatin is very elastic and opposite to ordinary photo-resist's brittleness. That is why Gelatin has been demonstrated as a protective mask in a sand-blasting process of glass plates [4]. Chemically, Gelatin is vulnerable to acid and base before cross-linking. Figure 7 demonstrates this with a sample coated with Gelatin, followed by standard photolithography (positive

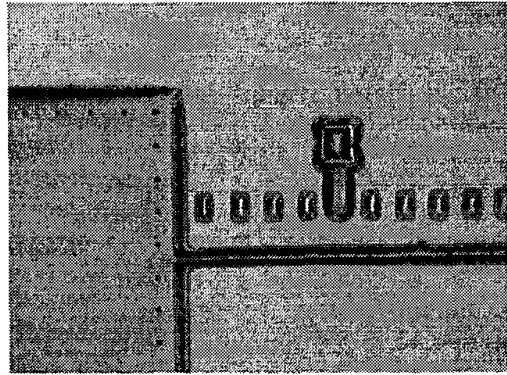

Fig. 5 Cross-linked Gelatin (20\%); thickness: 2.5 microns ; minimum line-width: 10 microns

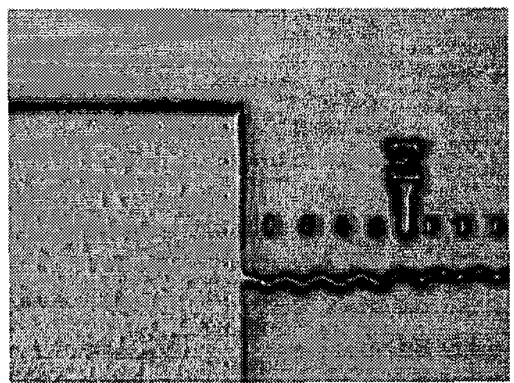

Fig. 6 Cross-linked Gelatin (32\%); thickness:6.5 microns ; minimum line-width: 10 microns

photo-resist over pure Gelatin layer.) When the top positive-resist is developed, the exposed Gelatin is attacked immediately by the alkaline developer (AZ 400K.) However, with the cross-linking using dichromate and UV light, the photo-patterned Gelatin (microns in thickness) becomes water-insoluble and chemical resistant to acids and bases. For example, Angus [5] reported that the Gelatin could be used as a gray-scale masking in electroplating some metallic samples. In addition, Gelatin is also plasma-resistant (easily more than hours.) Figures 8 and 9 show the surface of pure Gelatin after the dry-etching of $\mathrm{O}_{2}$ and $\mathrm{SF}_{6}$ plasma. The surface roughness seems to become worse, but the actual etching rate of the pure Gelatin is below $0.1 \mathrm{micron} / \mathrm{min}$ for $400 \mathrm{~W}$ of plasma (Technics PE-IIA Etcher.)

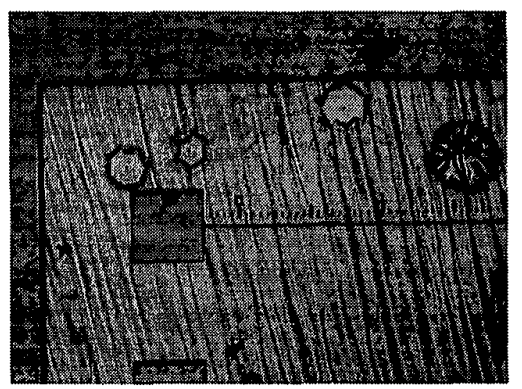

Fig. 7 AZ40OK attacks the surf ace of Gelatin (32\%) right after the developing of the above resist 


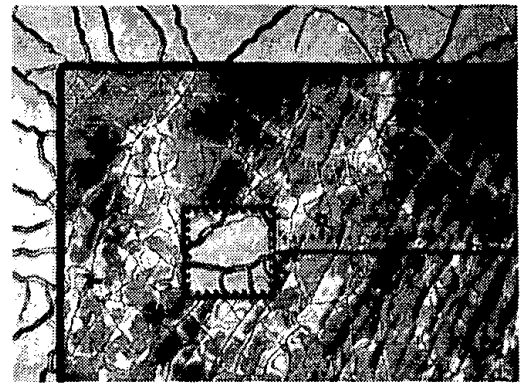

Fig. 8 Gelatin (32\%) surface after the etching of $\mathrm{O}_{2}$ plasma, cracks induced from the pure Gelatin underneath theAZ4620 positive resist

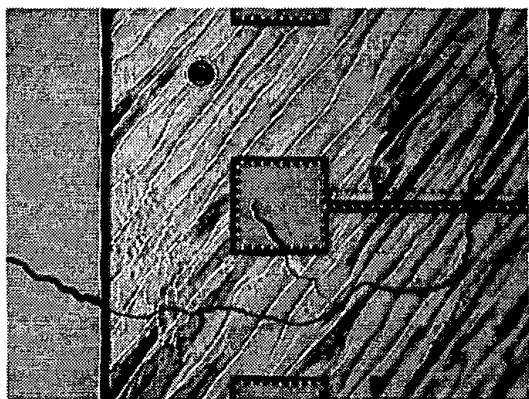

Fig. 9 Gelatin (32\%) surface after the etching of $S_{6}$ plasma, cracks induced from the pure Gelatin underneath theAZ4620 positive resist

\section{GELATIN USING AS A PROTECTION LAYER}

After surveying the preparation and properties of Gelatin films, we report here an example of using Gelatin as the protective and strengthening layers for large-size Parylene microfluidic MEMS structures. This idea is demonstrated in a process sketch of Fig. 10. By the use of the photo-patterned thick Gelatin (up to 10 microns or more), Parylene MEMS structures can be successfully made without stiction problems during the stripping of photo-resist sacrificial- layer using acetone. This is possible because the whole photolithographic patterning of Gelatin is a low temperature process, hence the Gelatin process does not destroy the already existing sacrificial photo -resist as well as the Parylene structures. Finally, the Gelatin protective layer could protect the weak Parylene structures from substrate-stiction during the resist-stripping because Gelatin is almost inert to acetone and to a variety of other organic solutions.

Figure 11 shows the substrate-stiction of the Parylene diaphragm due to the huge surface tension force of liquid inside. After we fabricated the thick, cross-linked Gelatin layer on the Parylene diaphragm, the whole stiffness did increase with one to two orders of magnitude higher. Figure 12 not only shows that the Parylene diaphragm does not stick to the substrate any more, but also the liquid inside the diaphragm changes to dry out from the center portion (whereas the case in Fig. 10 dries from the peripheral rim.)

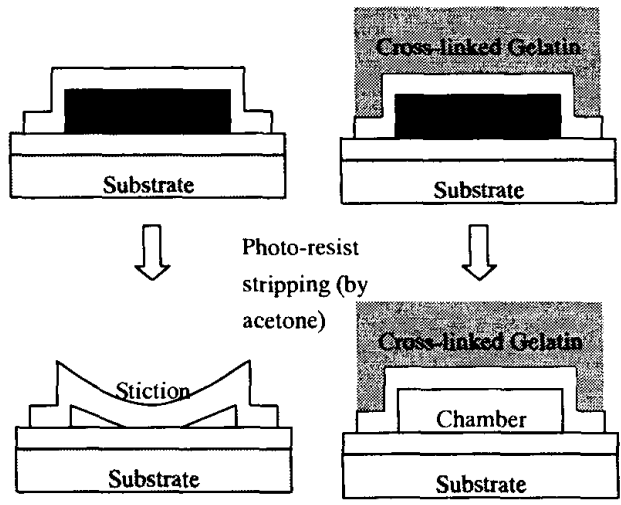

Fig. 10 The cross-section view of the weak microstructure (Parylene diaphragm) without Gelatin (left; stiction) and with Gelatin (right; no stiction)

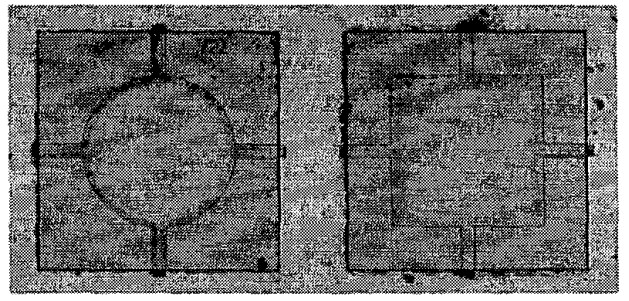

Fig. 11 The Parylene diaphragms of 3 microns thick sticking to the substrate after stripping the sacrificial photo-resist (also 3 microns high), the diameter or the width of the diaphragms is 500 microns.

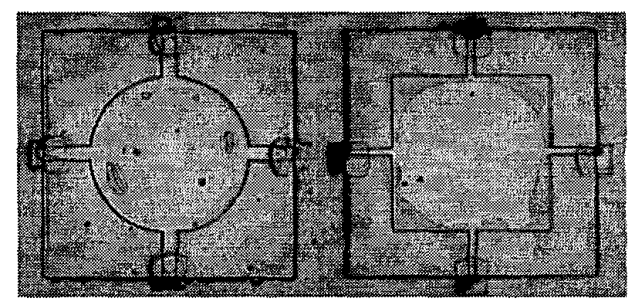

Fig. 12 The Parylene diaphragm covered with Gelatin of 10 microns thick not sticking to the substrate; coating speed of Gelatin: $500 \mathrm{rpm}$; concentration: $20 \% ; 1 \mathrm{~min} \mathrm{CF}_{4}$ plasma roughening the Parylene surface before Gelatin coating

\section{WATER-ABSORPTION, SWELLING EFFECT, AND ADHESION OF GELATIN}

By spin-coating, Gelatin can be deposited on the substrates with hydrophilic surfaces, e.g., glass substrates. There is no special treatment beside the general cleaning procedure for such substrates, and Gelatin adheres to it firmly after room temperature drying. The good adhesion does not change for the cross-linked Gelatin even suffering in acids or bases for hours.

However, we observed that Gelatin has tremendous trend of absorbing water and swells to one or two times la rger in size from its original pattems or geometry. The huge 
volumetric change of Gelatin makes the precise control of dimension fairly difficult, and even cause delamination of Gelatin film from the holding substrate.

Rinsing the cross-linked Gelatin in the ethanol alcohol could absorb the water molecules on the Gelatin surface quickly and is beneficial to the shape setting of Gelatin patterns. However, the sacrificial photo-resist under the Gelatin protective layer is vulnerable somewhat to ethanol. The rinsing time in the ethanol should be therefore controlled as short as possible.

On the other hand, although the cross-linked Gelatin is inert to acetone, yet the water inside Gelatin will be extracted by acetone and cause abrupt cracks everywhere. Such cracks not only degrade the surface morphology, but also induce afterward delamination of Gelatin with the substrate and make the protection layer unavailable during stripping the sacrificial photo-resist. Instead of rinsing in the ethanol solution, we hence re-rinsed the Gelatin with DI water to the ultimate situation of water-rich in this work before the photo-resist stripping.

Additionally, the poor adhesion between the Gelatin and Parylene films should be noted carefully. We enhanced the adhesion temporarily by means of roughening the Parylene surface using plasma etching. The recipe used for the fabrication of the sample shown in Fig. 12 is $100 \mathrm{~W} \mathrm{CF}_{4} / \mathrm{O}_{2}$ plasma for $1 \mathrm{~min}$ (SAMCO-RIE-10N.) The averaged roughness $\left(R_{A}\right)$ of the Parylene film before coating Gelatin is measured to be $90 \mathrm{~nm}$ by Alpha-Step 500 surface profiler.
An example of using Gelatin as the protective and strengthening layers for large-size Parylene microfluidic MEMS structures was demonstrated successfully in this paper. As far as we know, no other material/process can fulfill the same requirements, which makes Gelatin a useful material for MEMS fabrication. We believe Gelatin is useful for the MEMS community. Further research on this material will produce more interesting applications.

\section{ACKNOWLEDGEMENT}

The authors appreciate the financial support of this work from the National Science Council, Taiwan ROC, with the project number of NSC-89-2217-E-032-001. The authors also want to thank the helps from Mr. Yong $\mathrm{Xu}$ and Mr. T.N. Pornsin-Sirirak of the Micromachining group, Caltech, USA.

\section{REFERENCES}

[1] X.Q. Wang, et al., "Parylene micro check valve", Proceedings of the IEEE MEMS-1999, pp. 177-182, 1999.

[2] T.N. Pornsin-Sirirak, et al., "Flexible parylene actuator for micro adaptive flow control", Proceedings of IEEE MEMS-2001, pp. 511-514, 2001

[3] Website: www.gelita.com/DGF-english

[4] W.A. Little, "Microminiature refrigeration", Rev. Sci. Instrum., 55(5), pp.661-680, 1984.

[5] J.C. Angus, et al., "Controlled electroplating through Gelatin films", J. Electrochem. Soc., 133(6), pp.1152-1160, 1986.

\section{CONCLUSION}

